\title{
Production of regenerated silkworm silk fibers from aqueous dopes through straining flow spinning
}

Textile Research Journal 2019, Vol. 89(2I-22) 4554-4567 (C) The Author(s) 2019 Article reuse guidelines: sagepub.com/journals-permissions DOI: $10.1177 / 0040517519838050$ journals.sagepub.com/home/trj

SAGE

\author{
Rodrigo Madurga 1,2, Alfonso M Gañán-Calvo ${ }^{3}$, \\ Triana Mariscal ${ }^{1,2}$, Gustavo R Plaza ${ }^{1,2} \odot$, Gustavo V Guinea ${ }^{1,2,4}$, \\ Manuel Elices ${ }^{1,2}$ and José Pérez-Rigueiro ${ }^{1,2,4}$ (1)
}

\begin{abstract}
The possibility of spinning regenerated silkworm (Bombyx mori) fibers from a range of aqueous dopes by using a biomimetic approach based on straining flow spinning is explored in this work. It is found that spinning conditions can be established that allow fibers to be produced from environmentally friendly dopes with fibroin concentrations even one order of magnitude lower than that of the natural system. However, it is also found that the spinning process is favored and that the mechanical properties of the fibers are improved when dopes with higher fibroin concentration are employed. Since highly concentrated fibroin solutions in water are unstable, a stabilizing agent is required in order to obtain a spinnable dope at such large protein concentrations. $\mathrm{CaCl}_{2}$ is found to be an adequate stabilizing agent compatible with the straining flow spinning process. The optimization of the spinning parameters leads to the production of high-performance fibers with a work to fracture comparable to that of the natural material.
\end{abstract}

\section{Keywords}

silk, regenerated fibers, fibroin, biomimetics

The spinning of spider and silkworm silks offers an outstanding example of a practical process through which high-performance fibers can be efficiently produced. ${ }^{1}$ Silk fibers are spun from aqueous protein solutions without requiring the harsh physicochemical conditions common in the production routes of most artificial polymeric fibers, ${ }^{2}$ while reaching outstanding values of tensile strength and strain at break. This remarkable process relies on three basic principles that constitute a sound basis for the development of biomimetic spinning techniques. The first (A) involves the usage of building blocks (proteins) with a sequence compatible with a self-assembly process $^{3}$ and provided with molecular switches that control the transition from the liquid to the solid phase. ${ }^{4,5}$ The second (B) entails the creation of a physicochemical environment in terms of $\mathrm{pH}$ and salt concentration that determines the conformation of the protein through molecular switches. ${ }^{6}$ The third $(\mathrm{C})$ requires mechanical stresses to complete the formation of the solid fiber from the protein solution. ${ }^{7}$
In the natural process, these conditions are provided by the silk proteins and by the silk gland. Silk proteins (referred to as fibroins for silkworm silk and spidroins for spider silk) are characterized by a molecular weight in excess of $300 \mathrm{kDa}^{8}$ and by containing a large number of repetitions of a few short motifs of sequence. ${ }^{3,8}$ These motifs can assemble in three-dimensional structures known as $\beta$-nanocrystals, which result from the

'Centro de Tecnología Biomédica, Universidad Politécnica de Madrid, Spain

${ }^{2}$ Departamento de Ciencia de Materiales, ETSI Caminos, Canales y Puertos, Universidad Politécnica de Madrid, Spain

${ }^{3}$ Escuela Técnica Superior de Ingenieros, Universidad de Sevilla, Spain

${ }^{4}$ Biomedical Research Networking Center in Bioengineering, Biomaterials and Nanomedicine (CIBER-BBN), Spain

\section{Corresponding author:}

José Pérez-Rigueiro, Universidad Politécnica de Madrid, ETSI Caminos, Canales y Puertos, C/Professor Arangúren s/n Madrid, 28040 Spain. Email: jose.perez@ctb.upm.es 
piling-up of $\beta$-sheets. ${ }^{9,10}$ The formation of the $\beta$-nanocrystals is the critical step in the transition from the protein solution to the solid fiber. It is initiated by conformational changes that the $\mathrm{N}$ - and $\mathrm{C}$-terminal ends of the proteins undergo, ${ }^{4,5}$ which act as molecular switches, and is completed by the mechanical stresses to which the fiber is exposed along the duct of the silk gland. ${ }^{11}$ Such a process seems to be favored by the large protein concentrations (up to $50 \% \mathrm{w} / \mathrm{v}$ ) in the natural dope. ${ }^{12}$

The elegance and economy of the natural process has led to a number of different attempts that tried to produce regenerated silk fibers for a number of new applications. These attempts can be grouped into one of three possible approaches ${ }^{13}$ : wet spinning and related techniques, electrospinning and biomimetic spinning.

Wet spinning and related techniques (dry spinning and dry-jet wet spinning) are based on the removal of the solvent molecules from the dope, so that the protein-protein interactions lead to the formation of the solid fiber. In the wet spinning process the removal of the solvent molecules is the result of the interaction between the dope and a coagulating fluid. A similar approach is followed in the dry-jet wet spinning process, but the jet of the dope goes through an air gap before entering the coagulating bath. Lastly, dry spinning is based on the removal of the solvent molecules through the interaction of the dope with a gas flow.

It is assumed that the first description of a wet spinning process of regenerated silk fibers dates back to $1960,{ }^{14}$ in which an aqueous dope of fibroin was coagulated into a fiber by using a concentrated ammonium sulfate solution. Subsequent attempts relied on dopes that contained relatively harsh chemicals, such as orthophosphoric acid $\left(\mathrm{H}_{3} \mathrm{PO}_{4}\right){ }^{15}$ formic acid ${ }^{16,17}$ or hexafluoroacetate hydrate, ${ }^{18}$ among others. ${ }^{19-22}$ Usage of these solvents allowed high concentrations of silk protein in the solutions ${ }^{23}$ to be reached, which was considered suitable for the efficiency of the spinning process. In this regard, regenerated fibers spun from a highly concentrated fibroin solution and coagulated in methanol reached remarkable values of tensile strength $\left(\sigma_{u} \sim 400 \mathrm{MPa}\right)$ and strain at breaking $\left(\varepsilon_{u} \sim 40 \%\right)$ after being stretched in water. ${ }^{17}$ The benefits of a highly concentrated protein dope were also exhibited by the production of high-performance regenerated silk fibers from a highly concentrated dope stabilized with $\mathrm{N}$-methyl morpholine oxide (NMMO). ${ }^{24,25}$ In this case remarkable values of tensile strength of $\sigma_{u} \sim 400 \mathrm{MPa}$ and of strain at breaking of $\varepsilon_{u} \sim 30 \%$ were reached.

Alternatively, highly concentrated solutions were also prepared using hexafluoroisopropanol (HIFP) as the solvent ${ }^{26}$ or even from aqueous dopes following a procedure based on the slow increase of the protein concentration in the solution. ${ }^{27,28}$ This procedure led to fibers with values of work to fracture as high as $W_{f} \sim 80 \mathrm{MJ} / \mathrm{m}^{3}$ after being subjected to a post-spinning process in an atmosphere with high relative humidity. Lastly, protein concentrations in excess of $10 \% \mathrm{w} / \mathrm{v}$ were produced using $\mathrm{Ca}^{2+}$ as a stabilizer and led to the production of high-performance silk fibers through a dry spinning method, ${ }^{29}$ so that fibers with values of tensile strength of $\sigma_{u} \sim 300 \mathrm{MPa}$ and strain at breaking of $\varepsilon_{u} \sim 30 \%$ were obtained.

Electrospinning represents a conventional alternative to wet spinning and related techniques. The dope in an electrospinning process must possess such electrical properties that a fluid jet is formed and its flow is controlled by an intense electric field. The dope jet goes from its outlet to the opposite electrode and the solvent molecules are evaporated to the environment. ${ }^{30}$ The electrospinning of regenerated silk fibers allows the efficient spinning of non-wovens and mats, although the tensile properties of the individual electrospun fibers tend to be poorer than those of their wet spun counterparts. ${ }^{31-34}$

In addition to the conventional wet spinning and electrospinning processes, an enhanced understanding of the principles on which the natural spinning process is based has led to the development of a number of biomimetic techniques, many of which rely on the dependence of self-assembly of the silk proteins on the $\mathrm{pH}$ variations of the surrounding medium. ${ }^{35-39}$ These techniques have allowed reaching values of tensile strength as high as $\sigma_{u} \sim 600 \mathrm{MPa}^{35}$ and values of strain at breaking as high as $40 \%{ }^{39}$

Among the biomimetic processes, straining flow spinning (SFS) appears to be a versatile and robust technique that allows efficient spinning of regenerated silkworm silk fibers. ${ }^{40}$ In this regard, the versatility of SFS is the result of the large number of geometrical and hydrodynamic parameters, ${ }^{41}$ as well as of the chemistries of the coagulants ${ }^{42}$ that can be employed. Thus, compared with most wet spinning and electrospinning approaches, the inclusion of geometrical and hydrodynamic processing parameters as an integral part of the technique allows spinning under a much wider range of chemistries. In particular, it is shown that remarkable results are obtained while restricted only to aqueous-based, environmentally friendly chemistries. In addition, SFS allows overcoming the usual scalingup problems associated with other biomimetic processes proposed for the production of regenerated silk fibers.

In this context, this work continues exploring the possibilities of this biomimetic process by establishing a range of aqueous and environmentally friendly dopes compatible with the technique. In particular, it is shown that SFS allows working with protein concentrations as 
low as $4 \%(\mathrm{w} / \mathrm{v})$, although the production of high-performance fibers is favored by larger protein concentrations. The production of high-performance and biocompatible silk fibers ${ }^{43}$ opens up a wide range of novel applications in the field of biomaterials, including sutures, prostheses for tendons and ligaments and scaffolds in tissue engineering.

\section{Methods}

\section{Preparation of the dope}

Silkworm (Bombyx mori) silk cocoons were degummed in water by using an autoclave in a ratio $1 / 50(\mathrm{w} / \mathrm{v})$ at $121^{\circ} \mathrm{C}$ for 50 minutes. The degummed silk was dissolved in a $9.4 \mathrm{M} \mathrm{LiBr}$ solution and dialyzed against deionized water, as explained in detail elsewhere. ${ }^{40}$ The result after dialysis is an aqueous $3-4 \%(\mathrm{w} / \mathrm{v})$ fibroin solution.

In order to prepare dopes with higher fibroin concentrations, the solutions were concentrated by reverse dialysis (molecular weight cut-off, $3500 \mathrm{Da}$ ) for 15 hours against a polyethylene glycol (PEG) $8000 \mathrm{Da}$ solution in either deionized water or in a $1 \mathrm{M} \mathrm{CaCl}_{2}$ aqueous solution. When the reverse dialysis proceeded against the $1 \mathrm{M} \mathrm{CaCl}_{2}$ solution, it was considered that the final concentration of $\mathrm{CaCl}_{2}$ in the dope was $1 \mathrm{M}$, assuming that equilibrium was established between the dialysis medium and the dope at the end of the process. $\mathrm{CaCl}_{2}$ was added directly to the $4 \%$ fibroin solution $(4 \%-\mathrm{Ca})$, since reverse dialysis was not necessary for preparing solutions of this fibroin concentration. The addition of $\mathrm{CaCl}_{2}$ was essential for obtaining the $16 \%$ and $24 \%$ fibroin concentrations, since it was found that fibroin solutions without $\mathrm{CaCl}_{2}$ gel at concentrations of $\sim 10 \%$ (w/v). The final fibroin concentration was determined by the PEG concentration during the reverse dialysis. Fibroin concentrations of $8 \%, 16 \%$ and $24 \%$ were obtained by using PEG concentrations of $10 \%$, $20 \%$ and $30 \%$, respectively. The fibroin concentration of the dope was determined from the dry weight of a given volume of the dope solution and by taking into account the presence of $\mathrm{CaCl}_{2}$ in the dopes that contained this salt. It was found that the dope with the $24 \%$ concentration was unsuitable for spinning under the conditions used in this work, although it cannot be discarded that fibers might be obtained from such a concentrated dope by varying the SFS processing parameters adequately.

\section{Spinning parameters}

SFS requires establishing four sets of parameters, as explained in detail elsewhere ${ }^{40}$ : geometrical, hydrodynamic, composition of the coagulant and composition of the dope. The geometrical parameters were fixed to the following: diameter of the orifice of the nozzle, $D_{1} \sim 400 \mu \mathrm{m}$; inner diameter of the capillary, $d_{1}=150 \mu \mathrm{m}$; and tapering angle at the end of the capillary, $\alpha=90^{\circ}$. The hydrodynamic parameters include the flow rate of the dope, the flow rate of the focusing fluid and the velocity of the take-up roller. The flow rate of the dope was fixed at $Q_{d}=5 \mu \mathrm{l} / \mathrm{min}$ and the velocity of the take-up roller at $V_{R 1}=3 \mathrm{~m} / \mathrm{min}$ in all cases. The flow rate of the focusing fluid, $Q_{f}$, was optimized for each composition of the focusing fluid. The same composition was used for the focusing fluid and for the coagulating bath. Two different coagulant chemistries were selected according to the results obtained in a previous published work $^{42}$ : an aqueous PEG (average molecular weight $8000 \mathrm{Da}$ ) at a concentration of $30 \%$ $\mathrm{w} / \mathrm{v}$ (PEG30) solution, or a mixture of ethanol $80 \%$ and acetic acid in water at a final concentration of $0.2 \mathrm{M}$ acetic acid (Ac-Et80). These coagulants were selected for two reasons: (1) both coagulants are environmentally friendly and do not require any special safety requirements; and (2) a dehydrating component is included in each coagulant chemistry (e.g. PEG or ethanol). The addition of acetic acid to the Ac-Et 80 coagulant is based on the effect that a decrease in $\mathrm{pH}$ exerts on the fibroin assembly in the natural system. For each coagulant, the selection of the flow rate of the focusing fluid proceeded by determining the minimum and maximum values of $Q_{f}$ that allowed spinning at least $2 \mathrm{~m}$ of fiber without either leading to the breaking of the thread or to the accumulation of the fiber in the coagulating bath. Both of these values and their average were used for the different spinning processes. Under these conditions, the flow rates ranged from 1 to $5 \mathrm{ml} / \mathrm{min}$ for the Ac-Et80 coagulant and from 0.5 to $3.5 \mathrm{ml} / \mathrm{min}$ for PEG30.

Six different chemistries of the dope were tested: $4 \%$ fibroin in water $(4 \%) ; 8 \%$ fibroin in water $(8 \%) ; 4 \%$ fibroin in $1 \mathrm{M} \mathrm{CaCl} 2$ aqueous solution $(\mathrm{Ca}-4 \%)$; $8 \%$ fibroin in $1 \mathrm{M} \mathrm{CaCl}_{2}$ aqueous solution $(\mathrm{Ca}-8 \%)$; $16 \%$ fibroin in $1 \mathrm{M} \mathrm{CaCl}_{2}$ aqueous solution $(\mathrm{Ca}-$ $16 \%$ ); and $24 \%$ fibroin in $1 \mathrm{M} \mathrm{CaCl}_{2}$ aqueous solution (Ca-24\%).

From here on the spun fibers will be identified with a three-element code that will be used in the text to simplify the naming of the samples. The first part of the code indicates the composition of the dope, that is, $\mathrm{Ca}$ $4 \%$ for the $4 \%$ fibroin dissolved in $\mathrm{CaCl}_{2} 1 \mathrm{M}$. The second part of the code indicates the coagulant, that is, PEG for PEG 30\% aqueous solution and ET for the ethanol/acetic acid solution. Lastly, and when necessary, the third part specifies if the value of $Q_{f}$ used during spinning corresponds to the lowest (L), the highest $(\mathrm{H})$ or the average $(\mathrm{M})$ value of the flow rate of the focusing fluid. An example of a complete code is $\mathrm{Ca}-4 \%$-PEG-L, although in those cases in 
which the variation of $Q_{f}$ did not lead to significant changes in the properties of the fibers, the code will be shortened by removing the indication of the flow rate of the focusing fluid (i.e. Ca-4\%-PEG).

\section{Characterization of the regenerated fibers}

The microstructure of the regenerated silkworm silk fibers was analyzed by infrared spectroscopy and at least three Fourier transform infrared (FT-IR) spectra were obtained for each dope composition. Spectra were obtained by using a Nicolet iS 5 spectrometer equipped with an iD5 ATR complement, as described elsewhere. ${ }^{40}$ Each spectrum was obtained from a bundle of fibers and a background spectrum measured previously was subtracted from the experimental data. Spectra were determined as the average of 64 measurements in the range $550-4000 \mathrm{~cm}^{-1}$ (resolution $4 \mathrm{~cm}^{-1}$ ). The experimental amide I peak $\left(1580-1700 \mathrm{~cm}^{-1}\right)$ was fitted with Gaussian functions. The initial set of Gaussian functions was determined by following the criteria of the second derivative. The initial full width at half maximum (fwhm) was fixed at $8 \mathrm{~cm}^{-1}$. The fitting process was performed with Omnic 9 software. The assignment of the peaks was performed by following previous works ${ }^{42}$ and is summarized in Table 1.

The mechanical properties of the samples were characterized through tensile tests. At least six samples for each combination of dope and coagulating chemistries were tensile tested, so that at least two samples were tested for each value of $Q_{f}$. Consequently, some 60 samples were tested for each of the two coagulating chemistries.

Samples were mounted on aluminum foil frames with a gauge length of $20 \mathrm{~mm}$ which were fixed with ethylcyanocrylate. Before starting the tensile test, the diameter of the fibers was measured with an optical microscope (Leica DMI 3000B) by using the $40 \times$ objective. Then the aluminum frame was fixed to the upper and lower grips of an Instron 4411 tensile testing machine. The lower grip rested on a precision balance (Precisa XT220A, resolution $\pm 0.1 \mathrm{mgf}$ ) that was used for measuring force, as described elsewhere. ${ }^{44,45}$ After being mounted in the testing machine and determining the zero load point (the point at which no force is exerted on the fiber, but stresses build up immediately upon stretching), the initial length of the fiber, $L_{0}$, was measured with a caliper. Tensile tests were performed at a constant speed of $1 \mathrm{~mm} / \mathrm{min}$ and either in air (nominal environmental conditions of $25^{\circ} \mathrm{C}$ and $35 \%$ relative humidity) or immersed in water at $25^{\circ} \mathrm{C}$.

The cross-sectional area of the fibers was calculated from the median of the apparent diameters of all the fibers produced under the same spinning conditions,
Table I. Vibrational band assignment in the amide I region

\begin{tabular}{ll}
\hline Wavenumber $\left(\mathrm{cm}^{-1}\right)$ & Assignment \\
\hline $1594-1609$ & $\begin{array}{l}\text { Tyr side chain/aggregated strand } \\
\text { Aggregate } \beta \text {-strand/intermolecular } \\
\beta \text {-sheet }\end{array}$ \\
$1610-1620$ & $\begin{array}{l}\text { Intermolecular } \beta \text {-sheet } \\
1621-1627\end{array}$ \\
$1628-1637$ & Intramolecular $\beta$-sheet \\
$1638-1655$ & Random coil \\
$1656-1662$ & Helical structures \\
$1663-1694$ & $\beta$-turn \\
$1696-1703$ & Intermolecular $\beta$-sheet \\
\hline
\end{tabular}

assuming a circular cross-section. In the inhomogeneous samples the usage of the mean may be misleading, since it could give rise to an underestimation of the stresses that appear in the thinner sections of the fiber. The geometrical inhomogeneity of the samples was mostly related with the presence of PEG on the surface of the fibers coagulated with this chemistry, and usage of the median in this case is advisable, as explained above. Usage of the median in the more homogeneous fibers coagulated with the ethanol chemistry was preferred for allowing the direct comparison of all of the stress-strain curves presented in this work. Usage of the median instead of the mean does not induce significant changes in the values of the crosssectional area of more homogeneous samples.

Stresses were computed by using these cross-sectional areas and expressed in units of $\mathrm{MPa}$. Although conversion to alternative units such as gf/denier or $\mathrm{gf} /$ tex might be helpful for comparing the tensile properties of the regenerated fibers with other textile fibers, the difficulty in the measurement of density implies that the values would be affected by a relatively large degree of uncertainty. True stresses were calculated by assuming that the volume of the fiber remains constant during the entire testing process. ${ }^{46}$ After the tensile tests in air the samples were retrieved, metalized with gold for 45 seconds and observed by field-emission scanning electron microscopy (FE-SEM; Auriga Zeiss) at $V=5 \mathrm{kV}$ in order to obtain micrographs of the fracture surfaces.

\section{The wet-stretching process}

Wet-stretching consists of stretching the fiber up to a selected strain while immersed in water. ${ }^{47}$ In this work, fibers were typically stretched up to a value of 0.8 times the strain at break in water, unloaded and the distance between their ends fixed at the new zero load length. Water was then removed and the fibers were allowed to dry overnight before testing. 


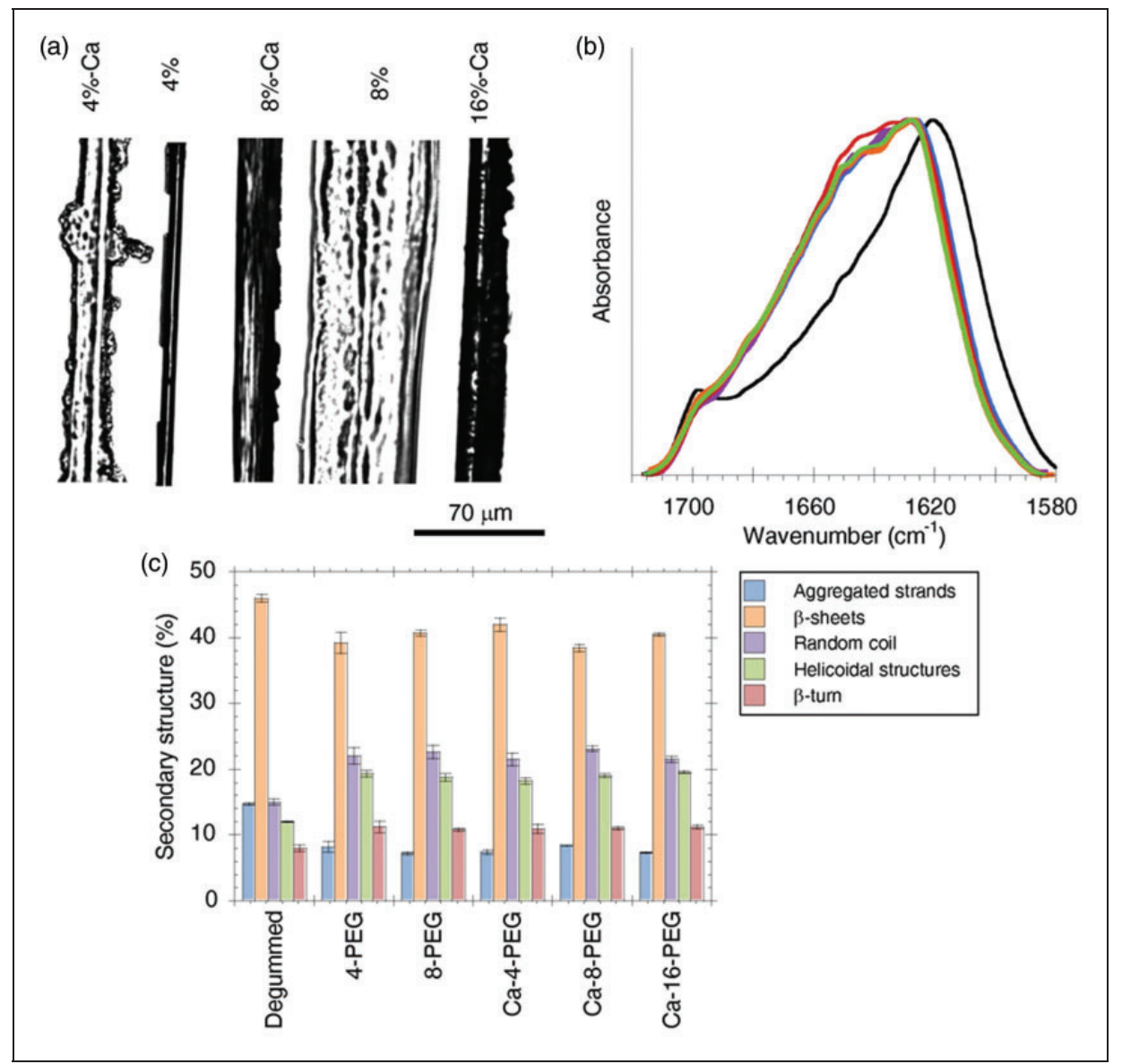

Figure I. Results obtained from the morphological and structural characterization of regenerated fibers coagulated with polyethylene glycol (PEG) 30\%. (a) Representative optical images of fibers spun from different fibroin dopes. (b) Representative attenuated total reflection-Fourier transform infrared spectroscopy amide I peak of fibers spun from dopes with fibroin concentration $4 \%$ (blue), $8 \%$ (red), Ca-4\% (purple), Ca-8\% (orange) and Ca-16\% (green). The spectrum of the degummed natural silkworm silk fiber (black) is shown for comparison purposes. (c) Contribution to the amide I peak of the different secondary structures in fibers spun from different fibroin dopes by using PEG as a coagulant. Error bars represent the standard error of three different spinning processes for each dope composition. (Color online only.)

\section{Results}

\section{PEG $30 \%$ coagulating bath}

Figure 1(a) shows representative optical images of the fibers spun using PEG $30 \%$ as the coagulant. There is a PEG coating around the fiber that remains even after the fiber is retrieved from the coagulating bath. The presence of the PEG coating makes the measurement of the diameter of the fiber difficult. For this reason, the values of the diameter used for calculating the crosssectional area were chosen from the apparently cleanest regions. However, a certain overestimation of the diameter and, consequently, an underestimation of the values of the stress cannot be discarded.

Figure 1(b) shows the amide I peak of fibers spun from dopes with different chemistries and coagulated with a PEG $30 \%$ aqueous solution. The amide I peak of natural degummed silkworm silk is also shown for comparison purposes. It is clear that there are no significant differences between the spectra of the regenerated fibers, although there is a clear shift of the maxima with respect to the spectrum of degummed natural silk. The deconvolution of the peaks (Figure 1(c)) reveals that such a shift is due to a higher content of random coil and helical structures in regenerated fibers. In contrast, natural silk shows a higher content in the more aligned $\beta$-sheet and aggregated strand ${ }^{48}$ conformations. The high concentration of $\mathrm{Ca}^{2+}$ in the dope of the $4 \%-\mathrm{Ca}, 8 \%-\mathrm{Ca}$ and $16 \%-\mathrm{Ca}$ samples implies that these fibers might contain a significant proportion of $\mathrm{Ca}^{2+}$ ions upon solidification. The concurrence of the FT-IR spectra of all samples in Figure 1(b), however, suggests that the presence of $\mathrm{Ca}^{2+}$ in the dope does not lead to any significant variation in the microstructure of the material. In addition, the specific analysis of 


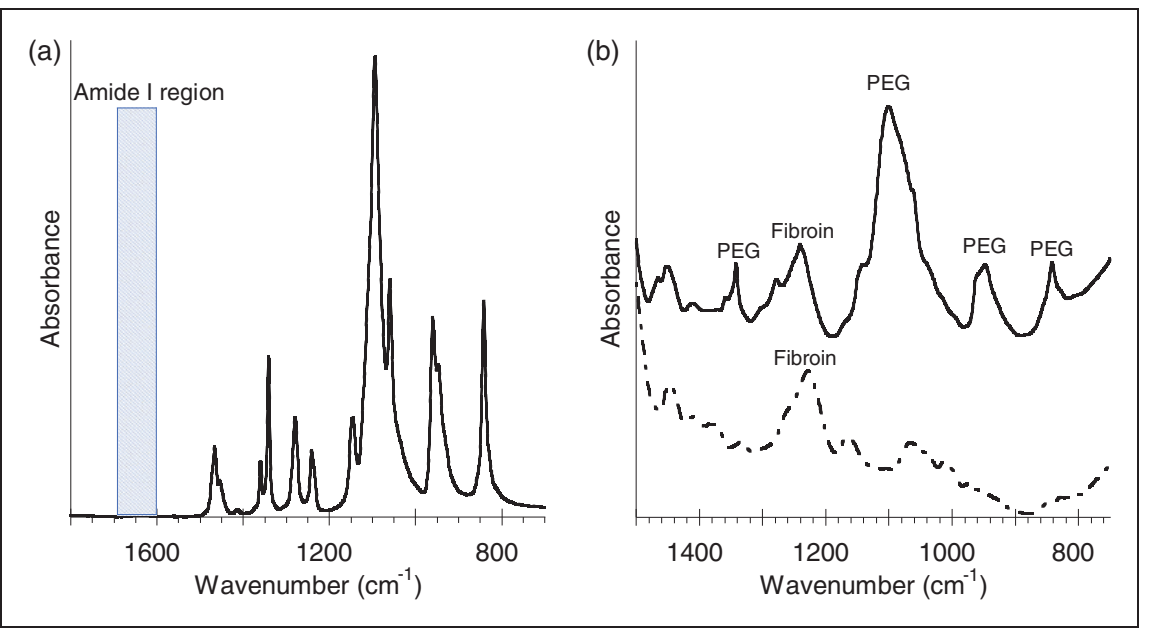

Figure 2. (a) Fourier transform infrared (FT-IR) spectra of polyethylene glycol (PEG) in which the region corresponding to the amide I peak is highlighted. (b) FT-IR spectra of an as-spun sample with a PEG coating (solid line curve) and after being immersed in water (broken line). The characteristic peaks of PEG and fibroin in this region of the spectrum are indicated.

regenerated SFS fibers by energy dispersive spectroscopy (EDS) could not detect the presence of calcium ions in the fiber. ${ }^{49}$ Although EDS is usually employed for the compositional characterization of surfaces, the technique has a depth resolution of the order of a few microns, which is comparable to the size of the fibers. Consequently, from the previous results it can be concluded that the $\mathrm{Ca}^{2+}$ ions initially present in the dope diffuse to the focusing fluid and to the coagulating bath during spinning.

The possible influence of the presence of PEG on the surface of the samples on their microstructural characterization was considered in a previous paper ${ }^{42}$ and the main results are summarized below. Figure 2(a) shows the FT-IR spectrum of PEG obtained under the same experimental conditions as those used for measuring the FT-IR of silk fibers. It is apparent that the FT-IR of PEG does not show any significant peak in the amide I region (1580-1750 $\left.\mathrm{cm}^{-1}\right)$, meaning that the deconvolution of this peak in the elementary secondary structures of the silk proteins is unaffected by the presence of the PEG coating. Figure 2(b) compares the region of FT-IR spectra of a sample coagulated with PEG before and after being washed in water. It is apparent that immersion in water leads to a significant reduction of the peak characteristic of PEG. However, since no significant differences in the mechanical properties of the fibers before or after being washed were observed, no systematic attempt at removing the PEG coating from the regenerated fibers previous to the characterization of the latter was performed.

Representative true stress-true strain curves obtained from tensile tests in air for each dope composition are shown in Figure 3(a). The mean and standard error of the tensile strength and strain at break are computed from all the samples spun using the same dope composition, except for the Ca8\%-PEG samples (see below). As indicated above, some samples were immersed in water to remove the PEG coating and no significant difference of these fibers with those of the controls with the PEG coating was observed. No clear correlation is found between the chemistry of the dope and the mechanical properties of the fibers. Samples $\mathrm{Ca}-4 \%$ and $8 \%$ show a brittle behavior, while samples $4 \%$, $\mathrm{Ca}-8 \%$ and $\mathrm{Ca}-16 \%$ show an incipient ductile behavior, with a strain at break-up of 0.18 . All samples show low breaking strengths of around $15 \mathrm{MPa}$. The samples Ca-8\%-PEG-L, however, represent a remarkable exception to this general trend, since these samples show an extremely high strain at break, which leads to a work to fracture of $W_{f}=37 \pm 4 \mathrm{MJ} / \mathrm{m}^{3}$. This value represents 0.6 of the work to fracture in natural silkworm silk fibers. ${ }^{45}$ Differences between the Ca- $8 \%$-PEG-L and the other fibers, including those produced from the same dope but with different values of $Q_{f}$, do not concern only its tensile properties. Ca- $8 \%$-PEG-L fibers show a corrugated pattern, as observed from SEM images (Figure 3(c)).

In previous works it was proved that the tensile testing of silk fibers in water offers a deep insight in some critical properties of the material. ${ }^{50}$ Tensile tests in water allow uncoupling the effects of the hydrogen bonds established between protein chains from other mechanisms responsible for the mechanical behavior of the fibers. Thus, the comparison of the tensile behavior of silk fibers in air and in water has allowed establishing models for the major ampullate gland silk, ${ }^{51,52}$ minor ampullate gland silk $^{53}$ and silkworm silk. ${ }^{54}$ Following this rationale, representative curves of the tensile tests in water for each dope composition are 


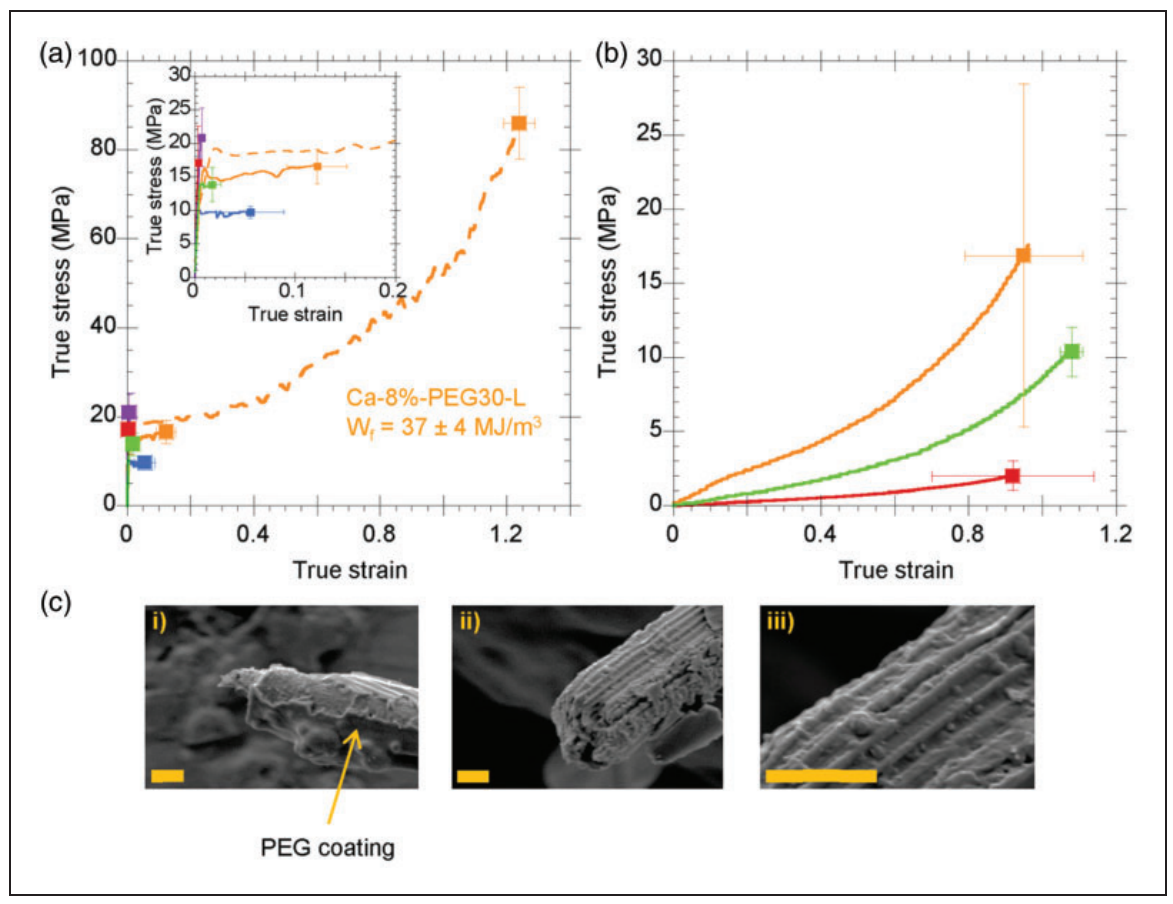

Figure 3. Results from the mechanical characterization of fibers coagulated with polyethylene glycol (PEG) $30 \%$. Representative true stress-true strain curves of fibers spun from the dopes: $4 \%$ (blue), $8 \%$ (red), Ca-4\% (purple), Ca-8\% (orange) and Ca- $16 \%$ (green) and tested in air (a) or in water (b). As stated in the text, Ca-8\%-PEG-L showed singular tensile properties and is not represented together with the $\mathrm{Ca}-8 \%$-PEG-H or $-\mathrm{M}$ data. Error bars represent the standard error of all samples spun from the same dope composition $(n \geq 6)$, except for the Ca-8\%-PEG-L samples, which correspond exclusively to the fibers spun with the lowest value of $Q_{f}$ under these conditions. (c) Representative scanning electron microscopy images of selected fibers: (i) lateral surface of a Ca- $4 \%$ fiber after fracture, in which the presence of an irregular PEG coating is observed; (ii) fracture surface of a Ca-8\%-PEG-L fiber; (iii) detail of the lateral surface of a Ca-8\%-PEG-L showing a corrugated pattern. Scale bars correspond to $3 \mu \mathrm{m}$.

presented in Figure 3(b). No results are shown for fibers spun from $4 \%$ and $\mathrm{Ca}-4 \%$ dopes because these fibers broke during the immersion in water. A significant increase in the strain at break of the samples when tested in water is observed, except for the $\mathrm{Ca}-8 \%$ PEG-L fibers. As shown in Figure 2(a), the Ca- $8 \%$ PEG-L reaches large values of strain at break, even when tested in air. Fibers spun from $\mathrm{Ca}-16 \%$ dopes show higher reproducibility in their tensile properties when compared with those spun from $8 \%$ or Ca- $8 \%$ dopes, as indicated by the shorter error bars.

\section{Ethanol $80 \%$ and acetic acid $0.2 \mathrm{M}$ coagulating bath}

Figure 4(a) shows optical images of the fibers spun by using the ethanol $80 \%$ and acetic acid $0.2 \mathrm{M}$ (Ac-Et80) coagulating bath. The measurements of the diameter of the fibers show that there is no change in the diameter for the dopes with $4 \%$ or $8 \%$ fibroin concentration (4\%: $\quad 13 \pm 2 \mu \mathrm{m}, \quad$ Ca- $4 \%$ : $\quad 11.5 \pm 0.3 \mu \mathrm{m}, \quad 8 \%$ : $12 \pm 1 \mu \mathrm{m}, \mathrm{Ca}-8 \%: 11.7 \pm 0.5 \mu \mathrm{m})$, but there is a slight increase in the diameter when the fibers are spun from the dope with $16 \%$ fibroin concentration (Ca-16\%: $14 \pm 1 \mu \mathrm{m})$. These results also show a higher reproducibility in the diameter of the fibers spun for the dopes that contain $\mathrm{CaCl}_{2}$.

Figure 4(b) shows the amide I peak of fibers spun from dopes with different chemistries and coagulated with Ac-Et80. The amide I peak of natural degummed silkworm silk is also shown for comparison purposes. In this case, the maxima of the amide I peak of the regenerated fibers concur with that of natural silk fibers. However, the spectra of regenerated fibers show a shoulder at around $1650 \mathrm{~cm}^{-1}$. The deconvolution of the amide I peaks (Figure 4(c)) indicates that all samples coagulated with Ac-Et80 have similar secondary structure contents.

On average, six samples of each dope composition were tensile tested in air, including two samples for each value of $Q_{f}$, with representative curves for each dope being shown in Figure 5(a). It is notable that the tensile strength of fibers spun from the Ca-16\% dope is almost double the value reached by the fibers spun from other dopes. The fractographic analysis of these fibers reveals a number of distinct features that, in some cases, could be correlated with the composition of the dope. Thus, fibers spun from dopes with either $4 \%$ or $8 \%$ fibroin concentration may show holes in the fracture surface 


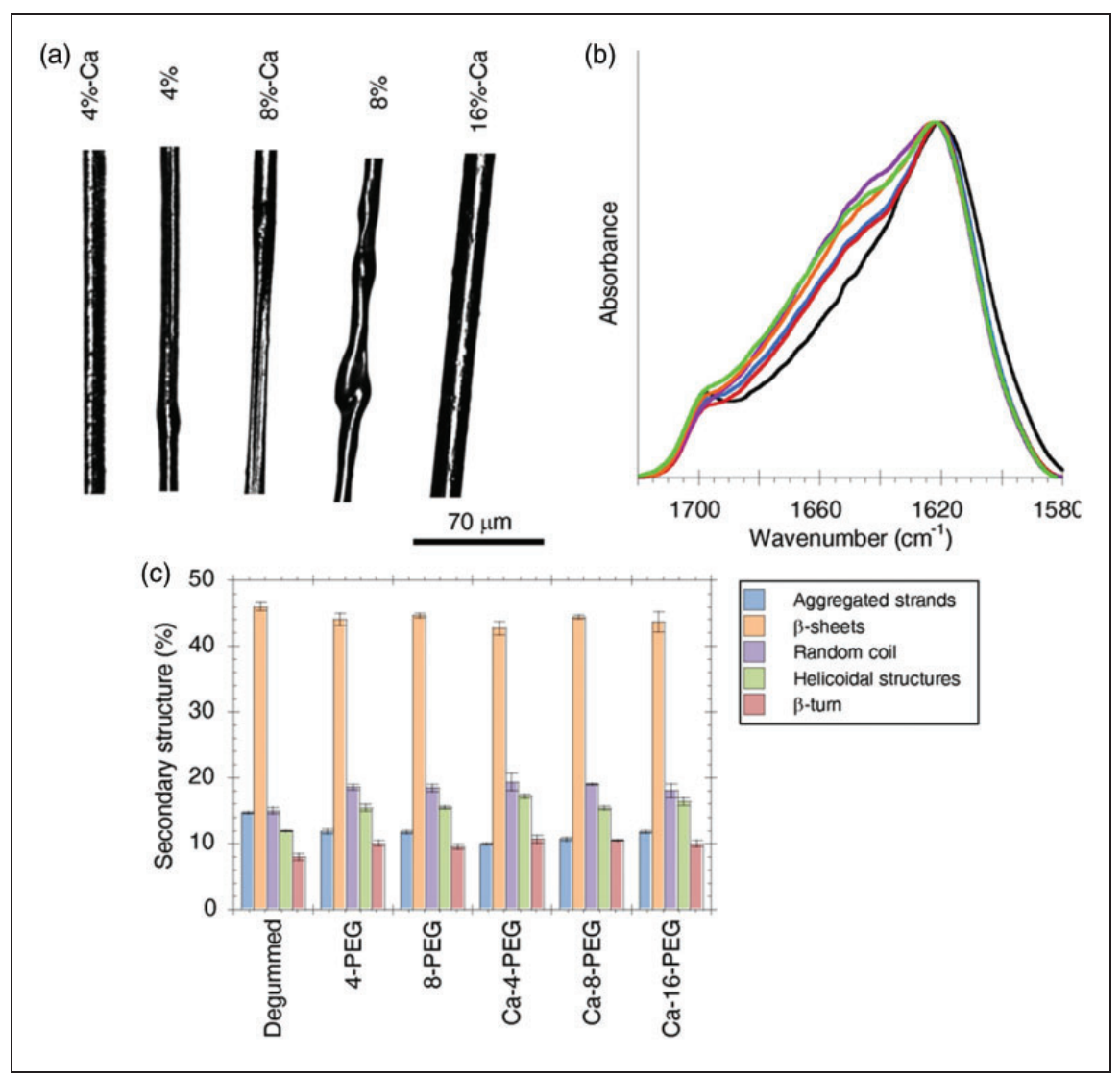

Figure 4. Results obtained from the morphological and microstructural characterization of fibers coagulated in a mixture of ethanol $80 \%$ and acetic acid in water at a final concentration of $0.2 \mathrm{M}$ acetic acid (Ac-Et80). (a) Representative optical images of fibers spun from different dopes. (b) Representative attenuated total reflection-Fourier transform infrared spectroscopy amide I peak of fibers spun from dopes with fibroin concentrations of $4 \%$ (blue), $8 \%$ (red), Ca-4\% (purple), Ca-8\% (orange) and Ca-16\% (green). The spectrum of the degummed natural silkworm silk fiber (black) is shown for comparison. (c) Contribution to the amide I peak of the secondary structures in fibers spun from different fibroin dopes using Ac-Et80 as the coagulant. Error bars represent the standard error of three spinning processes for each dope composition. (Color online only.)

(Figure 5(c)-(i)) or necking (Figure 5(c)-(ii)). Both features might be related to the reduced tensile strength of these fibers in comparison with those produced from the Ca$16 \%$ dope. These latter fibers showed flat and featureless fracture surfaces, as illustrated in Figure 5(c)-(iii).

Regardless of the dope composition, all the fibers coagulated in Ac-Et80 showed concurring true stresstrue strain curves when tested in water (Figure 5(b)). Compared with the stress-strain curves in air, all the fibers showed a significant increase in their strain at break. Only the tensile strength of the Ca- $16 \%$-ET samples seems to be affected in water, probably due to the relatively large value of tensile strength that these fibers reach when tested in air in comparison with the fibers spun under different conditions.

\section{Wet-stretching}

As shown in Figures 3(b) and 5(b), water has a plasticizing effect on the regenerated fibers. The influence of water on the tensile properties of the fibers is notable, since it was found in previous works ${ }^{47}$ that the tensile properties of regenerated silk fibers with a brittle behavior in air could be improved by stretching the fibers in water (i.e. fibers being subjected to a wetstretching process).

Figure 6(a) shows true stress-true strain curves obtained from fibers coagulated with PEG 30\% and subjected to wet-stretching. No data are shown for fibers spun from either $4 \%$ or $\mathrm{Ca}-4 \%$ dopes, given that those fibers broke while being immersed in water. A clear increase in the tensile strength and strain at break of the $8 \%$-PEG and Ca-16\%-PEG samples is observed when compared with the curves shown in Figure 3(a). In contrast, Ca-8\%-PEG-L samples, which showed notable tensile properties when tested in air, show an increase in tensile strength and a decrease in strain at break after being subjected to wet-stretching. Consequently, wet-stretching leads to an increase in the values of the work to fracture of 


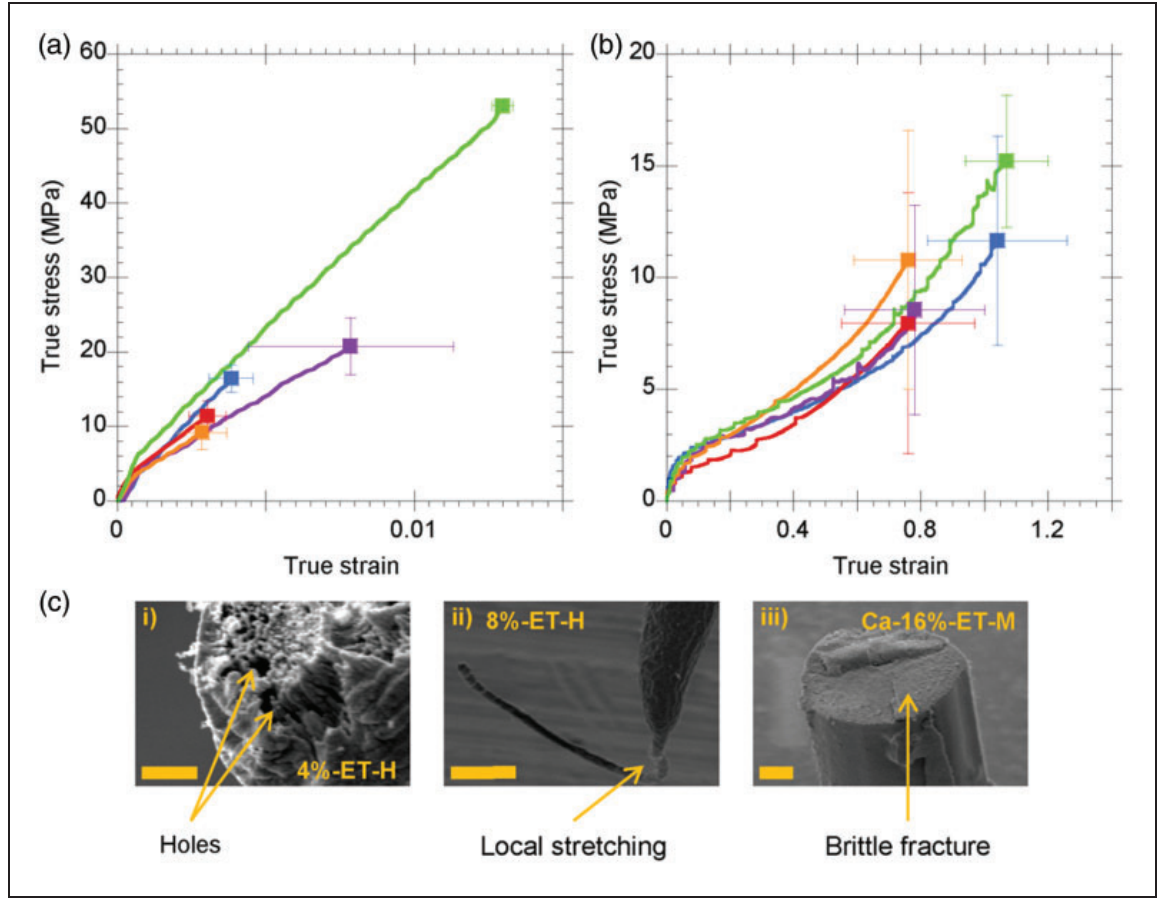

Figure 5. Results from the mechanical characterization of fibers coagulated in solution that is a mixture of ethanol $80 \%$ and acetic acid in water at a final concentration of $0.2 \mathrm{M}$ acetic acid. Representative true stress-true strain curves of fibers spun from dopes $4 \%$ (blue), $8 \%$ (red), Ca-4\% (purple), Ca-8\% (orange) and Ca-16\% (green) and tested in air (a) or in water (b). Notice the difference between the scales of the true strain axes of both figures. Error bars represent the standard error of all samples spun from the same dope composition $(n \geq 6)$. (c) Representative scanning electron microscopy images of the fracture surfaces of the fibers: (i) small holes were observed in the fibers with lower fibroin concentration (4\%); (ii) in additional necking might occur in fibers spun from fibroin dopes with a concentration lower than 10\%; (iii) flat fracture surfaces were found in the Ca-16\% dope fibers. Scale bars represent $3 \mu \mathrm{m}$. (Color online only.)

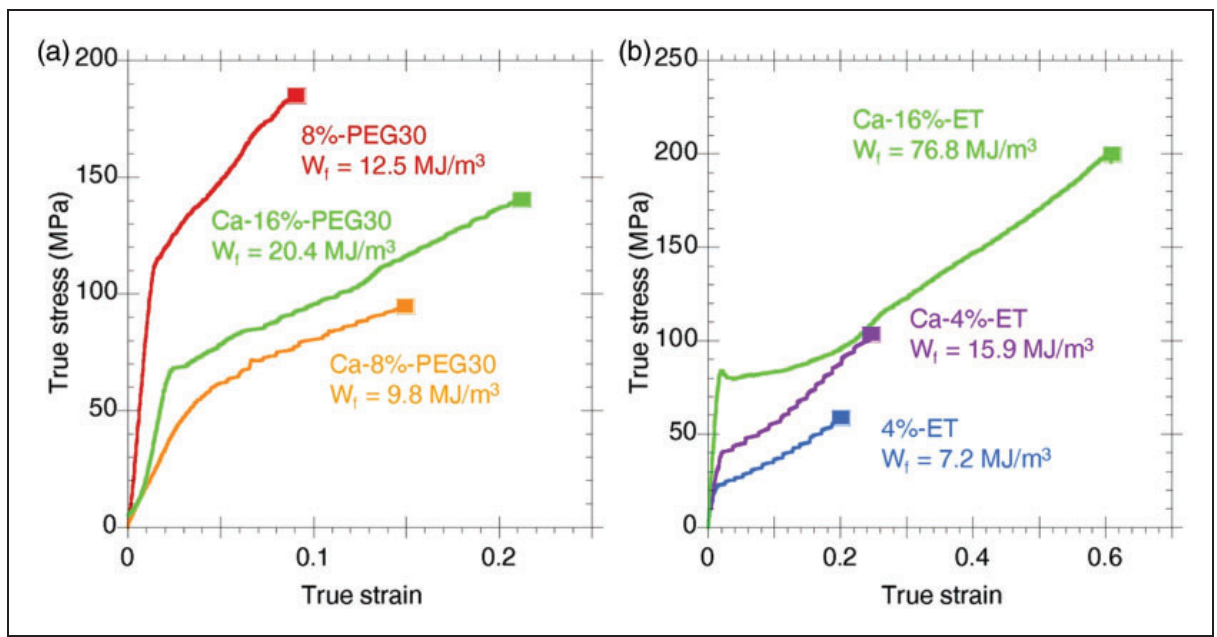

Figure 6. Results from the mechanical characterization of fibers coagulated with (a) polyethylene glycol (PEG) $30 \%$ and (b) Ac-Et80, after being subjected to a wet-stretching step. Fibers spun from dopes $4 \%$ (blue), $8 \%$ (red), Ca-4\% (purple), Ca- $8 \%$ (orange) and Ca-16\% (green) are presented.

the $8 \%$-PEG and $16 \%$-PEG samples, but reduces the work to fracture of $\mathrm{Ca}-8 \%$-PEG-L samples.

Figure 6(b) shows the true stress-true strain curves obtained from fibers coagulated in Ac-Et80 solution after being subjected to wet-stretching. In this case, no results were obtained for fibers spun from dopes with $8 \%$ fibroin concentration, as those fibers broke during the drying step after being stretched in water. 
The results show a consistent increase in the tensile strength and strain at break of the wet-stretched fibers. In this regard, the most notable improvement in the mechanical properties is observed for the Ca-16\%-ET condition, since a work to fracture of $W_{f} \sim 70 \mathrm{MJ} / \mathrm{m}^{3}$, comparable to that of natural silkworm silk, is obtained when the wet-stretched fiber is dried and tested in air.

\section{Discussion}

The results presented above show that SFS is compatible with a wide range of environmentally friendly aqueous dope chemistries and provides some hints on the influence of the composition of the dope on the spinning process and on the properties of the spun fibers.

\section{Range of fibroin concentrations compatible with SFS}

The data presented above show that SFS is compatible with aqueous dopes within a wide range of fibroin concentrations. In this regard, it is worth highlighting that versatility in terms of the dope concentration largely depends on the possibility of controlling the spinning process through the geometrical and hydrodynamic parameters. The influence of these sets of parameters in the process is shown, for instance, by the different ranges of focusing fluid flow rates required by the two different coagulating chemistries.

In the low-protein concentration limit it is established that fibers can be produced from dopes with a $4 \%(\mathrm{w} / \mathrm{v})$ concentration. This value of concentration is one order of magnitude lower than that of the natural spinning process, ${ }^{12}$ and ranges among the lowest fibroin concentrations from which it was reported that regenerated fibers can be spun from an aqueous dope. ${ }^{24,55-57}$ At the high-protein concentration limit, fibers were produced from dopes with $16 \%(\mathrm{w} / \mathrm{v})$ protein concentration. Dopes with even higher values of concentration were produced, but it was found that spinning with these dopes would require modifying the geometrical and/or hydrodynamic parameters employed in this work.

It was also found that aqueous fibroin solutions are unstable at concentrations higher than $8 \%$, meaning that the preparation of more concentrated solutions requires the addition of a stabilizing agent. $\mathrm{CaCl}_{2}$ was chosen as such a stabilizing agent due to the simplicity of adding this salt to the dope in comparison, for instance, with the procedure required to prepare aqueous silk dopes that contain NMMO. ${ }^{24}$ Stable solutions with a fibroin concentration from $4 \%$ to $24 \%$ were prepared by using $1 \mathrm{M} \mathrm{CaCl}_{2}$ aqueous solutions. Lowprotein concentration dopes $(4 \%$ and $8 \%)$ do not require the usage of $\mathrm{CaCl}_{2}$ to be stable, although Ca-containing dopes were prepared to assess the possible influence of $\mathrm{Ca}^{2+}$ ions in the dope on the microstructure and mechanical properties of the samples.

\section{Morphology and microstructure of the fibers}

The morphological and microstructural analysis of the fibers spun under different processing conditions allows the relative influence of the composition of the dope and of the coagulant on the properties of the fibers to be established. In this regard, the morphological analysis suggests a mild dependence of the fibroin concentration on the diameter of the fiber (higher concentrations lead to larger cross-sectional areas). In addition, the presence of calcium in the dope seems to increase the reproducibility of the diameter of the fibers. In spite of these relatively marginal influences, the diameter of the fiber seems to be mainly determined by the geometrical and hydrodynamic parameters of the process. ${ }^{41}$

The microstructural analysis of the regenerated fibers indicates that the coagulant seems to have a leading role with respect to the composition of the dope in determining the secondary structure of the proteins in the fibers. Thus, it is found that fibers coagulated in PEG reached an ordered fraction (calculated by adding the contributions of the $\beta$-sheets and aggregated strands) of $\sim 48 \%$, while those coagulated in ethanol/ acetic acid reach a value of $\sim 56 \%$. In addition, it is found that both values are largely independent of the precise composition of the dope. This result also indicates that the ordered fraction of the SFS regenerated fibers is lower than that found in natural silkworm silk, for which a value of $63 \%$ was estimated. ${ }^{58,59}$ These proportions of the ordered phase in the regenerated fibers suggest that an increase in the dehydrating capacity of the coagulant leads to higher values of the ordered phase, irrespective of the composition of the dope. However, it is also observed that FT-IR has a limited capacity to establish differences between samples spun with the same coagulating chemistry as shown, for instance, from the comparison of the spectrum obtained from the Ca-8\%-PEG-L sample and those of other samples coagulated with PEG. The limited resolution of FT-IR with respect to certain microstructural features of the fibers suggests the convenience of extending the microstructural analysis in future works to include other characterization techniques, such as nuclear magnetic resonance. ${ }^{60,61}$

\section{Mechanical behavior}

Two general trends are identified with regard to the mechanical behavior of the fibers from the results 
presented above. Firstly, tensile properties are more reproducible in samples spun from dopes with a higher protein concentration. This effect is particularly apparent from the error bars of the true stress-true strain curves corresponding to the $\mathrm{Ca}-16 \%$ samples shown in Figures 3 and 5. Secondly, the mechanical behavior of the as-spun regenerated fibers is always brittle, except for the Ca-8\%-PEG-L samples. Fibers spun under this condition reached a notable value of work to fracture, albeit still lower than that of the natural material.

In addition to these general trends, the previous results also point to subtler influences of the dope composition in combination with the other processing parameters on the properties of the fibers that require deeper analysis. Thus, the different focusing fluid flow rates used with different samples do not seem to exert a significant effect in most combinations of dope/ coagulant chemistries, as shown by the relatively small error bars presented in Figures 3 and 5. The samples Ca-8\%-PEG-L represent a notable exception to this general trend, since the properties of these fibers differ considerably from those of the Ca- $8 \%$ PEG-M and -H.

At this point, any discussion on the origin of this singular behavior in Ca-8\%-PEG-L samples must be necessarily speculative, although it might be argued that it might be related with two other peculiar features of these fibers: (1) the corrugated surface, as observed in Figure 3(c); and (2) the negative effect of wet-stretching on these samples, in comparison with the enhancement in the tensile behavior observed in the other fibers analyzed, as observed in Figure 6. Since the main effect of wet-stretching is an increase in the alignment of the protein chains, ${ }^{62}$ it can be hypothesized that the low focusing fluid rate and the relatively low fibroin concentration lead to a somewhat intermediate organization of the protein molecules in the fiber. This intermediate organization would allow the unfolding of the proteins ${ }^{63}$ when stretched in air, while being sufficient to sustain the stresses to which the fiber is subjected during tensile testing. The corrugated structure, which is characteristic of spinning processes in which there is a differential diffusion of the solvent from the dope ${ }^{64}$ might play a role in establishing this intermediate microstructure. Independently from its detailed origin, the exceptional behavior of Ca- $8 \%$-PEG-L proves that certain combinations of the processing parameters characteristic of the SFS technique allow reaching singular spots in the parametric space that yield fibers with peculiar properties.

Apart from the singular behavior of the $\mathrm{Ca}-8 \%$ PEG-L samples, the present study offers other examples of singular behaviors that depend on the precise combination of dope composition and the rest of the processing parameters. Such an example is offered, for instance, by the breaking of fibers spun from dopes with a fibroin concentration of $4 \%$ and coagulated in PEG30 when immersed in water. Another example is offered by the breaking of fibers spun from a fibroin concentration of $8 \%$ and coagulated in AcEt80 during the drying step after being immersed in water.

From an applied point of view, it is of utmost importance that the initially brittle tensile properties of the regenerated samples be modified through a wet-stretching process. ${ }^{49}$ Thus, it is found that even fibers spun from a $4 \%(\mathrm{w} / \mathrm{v})$ fibroin concentration dope reach a notable value of $20 \mathrm{MJ} / \mathrm{m}^{3}$ (one third of the work to fracture of the natural material) after being subjected to wet-stretching. However, as occurs with the overall homogeneity of the fibers, the best results are obtained from the more concentrated Ca-16\% dopes. These dopes allow the highest values of work to fracture with both coagulants after wet-stretching to be reached. In the case of Ca-16\%-ET samples, a value of $W_{f}=76 \mathrm{MJ} / \mathrm{m}^{3}$, comparable to that of natural silkworm silk, is obtained.

In summary, the previous analysis suggests, although some exceptions can be found depending on particular combinations of the processing parameters, that a higher protein concentration in the dope seems to favor the accretion and organization of the molecules in the solid fiber. This enhanced molecular assembly is consistent with the improved tensile behavior of the Ca-16\%-ET samples upon wet-stretching when compared with fibers produced from lower protein concentration dopes. The importance of the accretion of the proteins during the solidification of the protein is also suggested by the presence of voids in the fracture surface of samples spun from dopes with a protein concentration of $8 \%$ or lower. The presence of these voids points to an incorrect assembly of the material, probably related to a reduced mass flow from the lower protein concentration dopes. The presence of such defects might explain some characteristics that appear to be defined by certain combinations of the composition of the dope, the composition of the coagulant and the hydrodynamic parameters.

\section{Conclusion}

In this work it is shown that regenerated silkworm silk fibers can be spun efficiently through the biomimetic approach embodied by the SFS technique from a wide range of dope chemistries. In particular, the possibility of spinning regenerated silk fibers from aqueous and environmentally friendly dopes using SFS is established. In addition, it is shown that SFS allows spinning silk fibers from dopes within a wide range of fibroin 
concentrations. Thus, fibers are efficiently spun from dopes with a fibroin concentration of $4 \%(\mathrm{w} / \mathrm{v})$ to dopes with a fibroin concentration of $16 \%(\mathrm{w} / \mathrm{v})$. It is found, however, that fibroin solutions with concentrations higher than $8 \%(\mathrm{w} / \mathrm{v})$ are not stable, and require a stabilizing agent. In this regard, $\mathrm{CaCl}_{2}$ is shown to stabilize the highly concentrated dopes, while being totally compatible with the technique.

The availability of this wide range of dope chemistries is used to study the influence of the dope composition on the geometry, microstructure and mechanical properties of the material. Thus, a higher fibroin concentration leads to a slight increase in the diameter of the fibers, although the geometrical and hydrodynamic parameters also exert a significant influence on this feature. In contrast, the microstructural analysis reveals that the dope composition plays a secondary role with respect to the composition of the coagulant in the determination of the secondary structure of the proteins.

With respect to the mechanical behavior of the fibers, it is shown that almost all as-spun regenerated fibers showed a brittle behavior when tested in air, although a general trend was found that relates an enhanced tensile behavior with increasing fibroin concentration. This trend is more evident after subjecting the fibers to a wet-stretching process. In this case, values of work to fracture comparable to that of the natural material were measured in fibers spun from high-concentration fibroin dopes. It may be hypothesized that the usage of high-concentration dopes favors the mass flow from the solution to the solid fibers and leads to an enhanced organization of the protein molecules in the material.

Obtaining high-performance fibers with mechanical properties comparable to those of natural silk fulfils the biomimetic program for the production of regenerated silk fibers and opens a variety of new and promising applications for these materials, including, but not restricted to, the biomedical field. In particular, the availability of biocompatible silk fibers with remarkable mechanical properties offers the possibility of developing new scaffolds in tissue engineering that can sustain tensile loads, in contrast to most of the present solutions, which are basically designed to support compressive loads.

\section{Acknowledgments}

The authors wish to express their gratitude to Prof. José Luis Cenis (IMIDA-Spain), who kindly provided the silkworm cocoons.

\section{Declaration of conflicting interests}

The authors declared the following potential conflicts of interest with respect to the research, authorship, and/or publication of this article: The patent application of the SFS technology was filed on 18 December 2015. The SFS technology is licensed to Silk Biomed S.L. (Madrid, Spain).

\section{Funding}

The authors disclosed receipt of the following financial support for the research, authorship, and/or publication of this article: The work was supported by the Ministry of Economy and Competitiveness in Spain (Projects MAT2016-75544-C21-R, MAT2016-79832-R and DPI2016-78887-C3-1-R).

\section{ORCID iD}

Gustavo R Plaza (D) http://orcid.org/0000-0002-5555-5498

José Pérez-Rigueiro (D) http://orcid.org/0000-0001-8298-8398

\section{References}

1. Heim M, Keerl D and Scheibel T. Spider silk: from soluble protein to extraordinary fiber. Angew Chem Int Ed Engl 2009; 48: 3584-3596.

2. Hardy JG, Romer LM and Scheibel TR. Polymeric materials based on silk proteins. Polymer 2008; 49: 4309-4327.

3. Gatesy J, Hayashi C, Motriuk D, et al. Extreme diversity, conservation, and convergence of spider silk fibroin sequences. Science 2001; 291: 2603-2605.

4. Hagn F, Eisoldt L, Hardy JG, et al. A conserved spider silk domain acts as a molecular switch that controls fibre assembly. Nature 2010; 465: 239-242.

5. Askarieh G, Hedhammar M, Nordling K, et al. Selfassembly of spider silk proteins is controlled by a $\mathrm{pH}$ sensitive relay. Nature 2010; 465: 236-239.

6. Chen X, Shao ZZ and Vollrath F. The spinning processes for spider silk. Soft Matter 2006; 2: 448-451.

7. Iizuka E. Silk thread-mechanism of spinning and its mechanical-properties. Appl Polym Symposia 1985; 1985: 173-185.

8. Xia QY, Zhou ZY, Lu C, et al. A draft sequence for the genome of the domesticated silkworm (Bombyx mori). Science 2004; 306: 1937-1940.

9. Marsh RE, Corey RB and Pauling L. An investigation of the structure of silk fibroin. Biochim Biophys Acta 1955; 16: $1-34$.

10. Takahashi Y, Gehoh M and Yuzuriha K. Structure refinement and diffuse streak scattering of silk (Bombyx mori). Int J Biol Macromol 1999; 24: 127-138.

11. Ortlepp C and Gosline J. Consequences of forced silking. Biomacromolecules 2004; 5: 727-731.

12. Andersson $\mathbf{M}$, Johansson $\mathbf{J}$ and Rising A. Silk spinning in silkworms and spiders. Int $J$ Mol Sci 2016; 17: UNSP 1290.

13. Hoffmann B, Gruat-Henry C, Mulinti P, et al. Using hydrodynamic focusing to predictably alter the diameter of synthetic silk fibers. Plos One 2018; 13: e0195522.

14. Yazawa S. Spinning of concentrated aqueous silk fibroin solution. J Chem Soc Jpn 1960; 63: 1428-1430.

15. Ishizaka $\mathrm{H}$, Watanabe $\mathrm{Y}$, Ishida $\mathrm{K}$, et al. Regenerated silk prepared from ortho phosphoric acid solution of fibroin. J Ser Sci Jpn 1989; 58: 87-95. 
16. Wu HY, Zhang F, Yue XX, et al. Wet-spun silk fibroin scaffold with hierarchical structure for ligament tissue engineering. Mater Lett 2014; 135: 63-66.

17. Zhang F, Lu Q, Yue X, et al. Regeneration of high-quality silk fibroin fiber by wet spinning from $\mathrm{CaCl} 2$-formic acid solvent. Acta Biomaterialia 2015; 12: 139-145.

18. Yao JM, Masuda $\mathrm{H}$, Zhao $\mathrm{CH}$, et al. Artificial spinning and characterization of silk fiber from Bombyx mori silk fibroin in hexafluoroacetone hydrate. Macromolecules 2002; 35: 6-9.

19. Um IC, Kweon HY, Park YH, et al. Structural characteristics and properties of the regenerated silk fibroin prepared from formic acid. Int J Biol Macromol 2001; 29: 91-97.

20. Um IC, Kweon HY, Lee KG, et al. The role of formic acid in solution stability and crystallization of silk protein polymer. Int J Biol Macromol 2003; 33: 203-213.

21. Um IC, Kweon HY, Lee KG, et al. Wet spinning of silk polymer-I. Effect of coagulation conditions on the morphological feature of filament. Int J Biol Macromol 2004; 34: 89-105.

22. Um IC, Ki CS, Kweon HY, et al. Wet spinning of silk polymer-II. Effect of drawing on the structural characteristics and properties of filament. Int $J$ Biol Macromol 2004; 34: 107-119.

23. Matsumoto K, Uejima H, Iwasaki T, et al. Studies on regenerated protein fibers. 3. Production of regenerated silk fibroin fiber by the self-dialyzing wet spinning method. J Appl Polym Sci 1996; 60: 503-511.

24. Marsano E, Corsini P, Arosio C, et al. Wet spinning of Bombyx mori silk fibroin dissolved in N-methyl morpholine $\mathrm{N}$-oxide and properties of regenerated fibres. Int $J$ Biol Macromol 2005; 37: 179-188.

25. Plaza GR, Corsini P, Marsano E, et al. Old silks endowed with new properties. Macromolecules 2009; 42: 8977-8982.

26. Koyanagi R, Zhu Z and Asakura T. Regenerated Bombyx mori silk fiber with enhanced biodegradability. J Insect Biotechnol Sericol 2010; 79: 27-30.

27. Zhou GQ, Shao ZZ, Knight DP, et al. Silk fibers extruded artificially from aqueous solutions of regenerated Bombyx mori silk fibroin are tougher than their natural counterparts. Adv Mater 2009; 21: 366-370.

28. Yan JP, Zhou GQ, Knight DP, et al. Wet-spinning of regenerated silk fiber from aqueous silk fibroin solution: discussion of spinning parameters. Biomacromolecules 2010; 11: 1-5.

29. Yue X, Zhang F, Wu H, et al. A novel route to prepare dry-spun silk fibers from $\mathrm{CaCl} 2$-formic acid solution. Mater Lett 2014; 128: 175-178.

30. Jin HJ, Fridrikh S, Rutledge GC, et al. Electrospinning Bombyx mori silk with poly(ethylene oxide). Abs Pap Am Chem Soc 2002; 224: U431-U431.

31. Kim SH, Nam YS, Lee TS, et al. Silk fibroin nanofiber. Electrospinning, properties, and structure. Polym J 2003; 35: 185-190.

32. Zhu J, Zhang Y, Shao H, et al. Electrospinning and rheology of regenerated Bombyx mori silk fibroin aqueous solutions: the effects of $\mathrm{pH}$ and concentration. Polymer 2008; 49: 2880-2885.
33. Solanas C, Herrero S, Dasari A, et al. Insights into the production and characterization of electrospun fibers from regenerated silk fibroin. Eur Polym J 2014; 60: 123-134.

34. Aznar-Cervantes SD, Vicente-Cervantes D, MeseguerOlmo L, et al. Influence of the protocol used for fibroin extraction on the mechanical properties and fiber sizes of electrospun silk mats. Mater Sci Eng C Mater Biol Appl 2013; 33: 1945-1950.

35. Luo J, Zhang L, Peng Q, et al. Tough silk fibers prepared in air using a biomimetic microfluidic chip. Int $J$ Biol Macromol 2014; 66: 319-324.

36. Tokareva O, Jacobsen M, Buehler M, et al. Structurefunction-property-design interplay in biopolymers: spider silk. Acta Biomaterialia 2014; 10: 1612-1626.

37. Heidebrecht A, Eisoldt L, Diehl J, et al. Biomimetic fibers made of recombinant spidroins with the same toughness as natural spider silk. Adv Mater 2015; 27: 2189-2194.

38. Anton AM, Heidebrecht A, Mahmood N, et al. Foundation of the outstanding toughness in biomimetic and natural spider silk. Biomacromolecules 2017; 18: 3954-3962.

39. Andersson M, Jia Q, Abella A, et al. Biomimetic spinning of artificial spider silk from a chimeric minispidroin. Nat Chem Biol 2017; 13: 262-264.

40. Madurga R, Ganan-Calvo AM, Plaza GR, et al. Production of high performance bioinspired silk fibers by straining flow spinning. Biomacromolecules 2017; 18 : 1127-1133.

41. Madurga R, Guinea GV, Elices M, et al. Straining flow spinning: Simplified model of a bioinspired process to mass produce regenerated silk fibers controllably. Eur Polym J 2017; 97: 26-39.

42. Madurga R, Ganan-Calvo AM, Plaza GR, et al. Straining flow spinning: production of regenerated silk fibers under a wide range of mild coagulating chemistries. Green Chem 2017; 19: 3380-3389.

43. Vepari C and Kaplan D. Silk as a biomaterial. Progr Polym Sci 2007; 32: 991.

44. Perez-Rigueiro J, Viney C, Llorca J, et al. Silkworm silk as an engineering material. J Appl Polym Sci 1998; 70: 2439-2447.

45. Perez-Rigueiro J, Viney C, Llorca J, et al. Mechanical properties of single-brin silkworm silk. J Appl Polym Sci 2000; 75: 1270-1277.

46. Guinea GV, Perez-Rigueiro J, Plaza GR, et al. Volume constancy during stretching of spider silk. Biomacromolecules 2006; 7: 2173-2177.

47. Plaza GR, Corsini P, Perez-Rigueiro J, et al. Effect of water on Bombyx mori regenerated silk fibers and its application in modifying their mechanical properties. J Appl Polym Sci 2008; 109: 1793-1801.

48. Jackson $\mathbf{M}$ and Mantsch HH. The use and misuse of FTIR spectroscopy in the determination of protein-structure. Crit Rev Biochem Mol Biol 1995; 30: 95-120.

49. Madurga R, Gañán-Calvo AM, Plaza GR, et al. Comparison of the effects of post-spinning drawing and wet stretching on regenerated silk fibers produced through straining flow spinning. Polymer 2018; 150: 311-317. 
50. Work RW. Dimensions, birefringences, and force-elongation behavior of major and minor ampullate silk fibers from orb-web-spinning spiders-effects of wetting on these properties. Text Res $J$ 1977; 47: 650-662.

51. Termonia Y. Molecular modeling of spider silk elasticity. Macromolecules 1994; 27: 7378-7381.

52. Termonia Y. Molecular modelling of the stress/strain behaviour of spider dragline. In: Elices M (ed.) Structural biological materials. Amsterdam: Pergamon Press, 2000, pp.335-349.

53. Guinea GV, Elices M, Plaza GR, et al. Minor Ampullate silks from nephila and argiope spiders: tensile properties and microstructural characterization. Biomacromolecules 2012; 13: 2087-2098.

54. Perez-Rigueiro J, Viney C, Llorca J, et al. Mechanical properties of silkworm silk in liquid media. Polymer 2000; 41: 8433-8439.

55. Liivak O, Blye A, Shah N, et al. A microfabricated wetspinning apparatus to spin fibers of silk proteins. Structure-property correlations. Macromolecules 1998; 31: 2947-2951.

56. Kim HJ and Um IC. Effect of degumming ratio on wet spinning and post drawing performance of regenerated silk. Int J Biol Macromol 2014; 67: 387-393.

57. Chung DE and Um IC. Effect of molecular weight and concentration on crystallinity and post drawing of wet spun silk fibroin fiber. Fiber Polym 2014; 15: 153-160.
58. Sinsawat A, Putthanarat S, Magoshi Y, et al. X-ray diffraction and computational studies of the modulus of silk (Bombyx mori). Polymer 2002; 43: 1323-1330.

59. Belen Perea G, Solanas C, Mari-Buye N, et al. The apparent variability of silkworm (Bombyx mori) silk and its relationship with degumming. Eur Polym $J$ 2016; 78: 129-140.

60. Asakura T, Isobe K, Aoki A, et al. Conformation of crystalline and noncrystalline domains of [3-C-13]Ala-, [3-C-13]Ser-, and [3-C-13]Tyr-Bombyx mori silk fibroin in a hydrated state studied with C-13 DD/MAS NMR. Macromolecules 2015; 48: 8062-8069.

61. Nishimura A, Matsuda H, Tasei Y, et al. Effect of water on the structure and dynamics of regenerated [3-C-13] Ser, [3-C-13], and [3-C-13] Ala-Bombyx mori silk fibroin studied with C-13 solid-state nuclear magnetic resonance. Biomacromolecules 2018; 19: 563-575.

62. Guinea GV, Elices M, Perez-Rigueiro J, et al. Stretching of supercontracted fibers: a link between spinning and the variability of spider silk. J Exp Biol 2005; 208: 25-30.

63. Belen Perea G, Solanas C, Plaza GR, et al. Unexpected behavior of irradiated spider silk links conformational freedom to mechanical performance. Soft Matter 2015; 11: 4868-4878.

64. Chawla KK. Fibrous materials. Cambridge: Cambridge University Press, 1998. 